\title{
Minimally invasive aortic valve replacement with central cannulation: A cost-benefit analysis in a developing country
}

\author{
Mohammed Sanad ${ }^{1,2}$ and Hatem Beshir ${ }^{1,3^{*}}$ (D)
}

\begin{abstract}
Background: Minimally-invasive approaches to aortic valve replacement (MIAVR) are technically and logistically demanding. However, few centers have started using these approaches with standard equipment because of the limited resources. We sought to report intra- and postoperative clinical outcomes and address health resource utilization after MIAVR.

Results: A total of 102 eligible patients who had aortic valve replacement were enrolled in a prospective, multicenter cohort study conducted from June 2015 to December 2017. Fifty patients underwent aortic valve surgery via upper inverted T-shaped hemi-sternotomy (MS), and 52 patients were operated using full sternotomy (FS) in two centers in a developing country. Central cannulation was performed in all cases. Major adverse cardiac events, pain, and wound complications were compared. A cost analysis was performed, and exposure and feasibility for cannulation were assessed. The mean length of MS skin incision was $5.82 \pm 0.67 \mathrm{~cm}$. Cumulative cross-clamp time was insignificant between both groups $(91.87 \pm 34.41$ versus $94.91 \pm 33.96 \mathrm{~min} ; p=0.66)$. MS exhibited shorter ventilation time (6.18 \pm 1.86 versus $10.68 \pm 12.78 \mathrm{~h} ; p=0.029)$ and intensive care stays (33.27 \pm 19.75 versus $49.42 \pm 47.1 \mathrm{~h} ; p=0.037$ ). Major adverse cardiac events (MACEs) were compared, and MS group exhibited fewer transfusions ( $1.18 \pm 0.89$ versus $1.7 \pm 0.97$ units; $p=0.002$ ), fewer pulmonary complications ( 1 (2\%) versus 2 (3.8\%); $p$ $<0.001$ ), and less sternotomy wound infection (1 (2\%) versus $5(9.6 \%) ; p=0.048)$. Total operative mortality of $4.46 \%$ was recorded $(n=5)$. Significant cost reduction was recorded favoring MS; central cannulation saved $\$ 907.16$ and carried a total cost reduction of $\$ 580$ (9.3\%) when compared with the FS approach $(p<0.0001)$.

Conclusions: With a lack of logistics in developing countries, MIAVR not only has a cosmetic advantage but carries a significant reduction in blood use, respiratory complications, pain, and cost. MIAVR can be feasible, with a rapid learning curve in developing centers.
\end{abstract}

Keywords: Aortic valve replacement, Minimally invasive cardiac surgery, Mini-sternotomy, Central cannulation

\footnotetext{
*Correspondence: hatem.beshir@alexmed.edu.eg; hatem_beshir@msn.com Previous presentationsPresented as a poster presentation D38 during the proceedings of the 18th annual meeting of the international society of the minimally invasive cardiothoracic surgery (ISMICS 18), June 2018 in

Vancouver, Canada. Presented as a poster presentation \#470 during the proceedings of the 26th annual meeting of the Asian Society for Thoracic and Cardiovascular Surgery (ASCVTS 18), May 2018 in Moscow, Russia. Results Presented orally at the 15th annual congress of update in cardiology and cardiovascular surgery (UCCVS 2019), March 2019 in Antalya, Turkey.

'Department of Cardiothoracic Surgery, Faculty of Medicine, Mansoura University Hospitals, D17, F5. 60, El Gomhoria Street, Qism 2, Mansoura, Dakahlia 35516, Egypt

${ }^{3}$ Department of Cardiothoracic Surgery, Egypt Ministry of Health and

Population, Alexandria Directorate, Alexandria, Egypt

Full list of author information is available at the end of the article
} 


\section{Background}

In developing countries, rheumatic heart disease remains the leading cause of aortic valve disease, and surgical aortic valve replacement (SAVR) through full sternotomy is the standard approach [1].

Several minimally invasive techniques for cardiac surgery had been presented to our country since 2011 to lessen the intrusiveness of the surgical procedures, reduce pain, and shorten hospital stays while sustaining equivalent quality and safety of SAVR with a better quality of life and few complications as compared with conventional SAVR while maintaining cost-effectiveness.

Multiple impediments face cardiac surgery in developing nations. The lack of subsidy, high expenses linked to surgical equipment, and lengthier training curve make minimally invasive SAVR difficult as a standard of care and place a burden on the healthcare systems.

Multiple techniques for minimal invasive SAVR were proposed to decrease the length of skin incision and sternal involvement. Upper J-shaped sternotomy, inverted T-shaped mini-sternotomy, C-shaped sternotomy, and right anterior thoracotomy (RAT) approaches were described [2].

Two strategies of arterial cannulation were proposed: the retrograde route with femoral arterial cannulation and the antegrade route via direct ascending aorta cannulation. Cannulation of the groin was designated for refining exposure during surgery, but irrespective of the higher cost, in some instances, this tactic may lead to infection, lymphoid fistula, arterial wall dissection, distal limb ischemia, and increased risk of neurological complications [3].

The aim of this study was to compare the surgical outcomes and cost-effectiveness of SAVR with central cannulation using mini-sternotomy (MS) to full sternotomy in a developing country without additional equipment.

\section{Methods}

This prospective multicenter cohort study was conducted between June 2015 and December 2017 and included 112 adult patients who underwent surgical aortic valve replacement in 2 institutions. We followed the declaration of Helsinki regarding studies on human subjects [4].

The patients were operated by two surgical teams who performed surgery in both centers. Both surgical teams were oriented with conventional and minimally invasive surgical approaches.

There were 102 eligible cases who underwent isolated aortic valve replacement. Cases with valve calcifications and chronic endocarditis were included. Re-operative cases, emergency operation, patients with thoracic deformities, low ejection fraction (EF) (less than 40\%), active infective endocarditis, porcelain aorta, and
EuroSCORE II predicted mortality more than 10\%, and those who had concomitant cardiac surgery were excluded to create two equal groups. Two years of followup were conducted, and the vital status was reported.

Twelve patients were excluded from our sample; two cases were excluded from the MS group for concomitant ascending aorta replacement, and ten cases were excluded from the FS group; three had re-operative SAVR, and 7 cases had a concomitant ascending aorta replacement.

The MS group included 50 patients, and the FS group had 52 patients. We analyzed the operative, short-term hospital outcomes, mid-term outcomes, and costeffectiveness analysis of each technique.

\section{Preoperative stratification}

Computerized tomography (CT) was performed preoperatively to all cases to assess the aortic root position, the alpha angle, and the sternal to root distance. Candidate patients for the mini-sternotomy approach required more than $50 \%$ of the aortic root behind the sternum [2]. CT is of crucial importance for the exclusion of porcelain aorta and has a better role in stratification of cases eligible for the right anterior mini-thoracotomy approach.

The patients were stratified into two groups according to their definitive surgical route based on surgeons' preference:

1. Mini-sternotomy (MS) included 50 patients in whom SAVR was done via upper inverted T-shaped hemi-sternotomy.

2. Full sternotomy (FS) included 52 patients with conventional SAVR using full median sternotomy.

\section{Anesthetic protocol}

Under full invasive monitoring, the anesthetic protocol encompassed induction with fentanyl (1-10 $\mu \mathrm{g} / \mathrm{kg})$, atracurium besylate $(0.4-0.5 \mathrm{mg} / \mathrm{kg})$ for muscle relaxation, and propofol infusion $(100-300 \mu \mathrm{g} / \mathrm{kg} / \mathrm{h})$ for the maintenance of anesthesia to endorse early extubation. Pain medication included pethidine hydrochloride in the intensive care unit (ICU) and oral acetaminophen (500 mg) while in the ward.

\section{Surgical technique}

Following general anesthesia, the FS group had a full sternotomy, while the MS group underwent a 5-7 cm upper sternotomy skin incision followed by a $10 \mathrm{~cm}$ upper inverted $\mathrm{T}$ hemi-sternotomy using a primary sternal blade for longitudinal osteotomy and a redo blade for the transverse sternotomy [2] at the level of the third intercostal space. The conventional group had a classical full median sternotomy. 


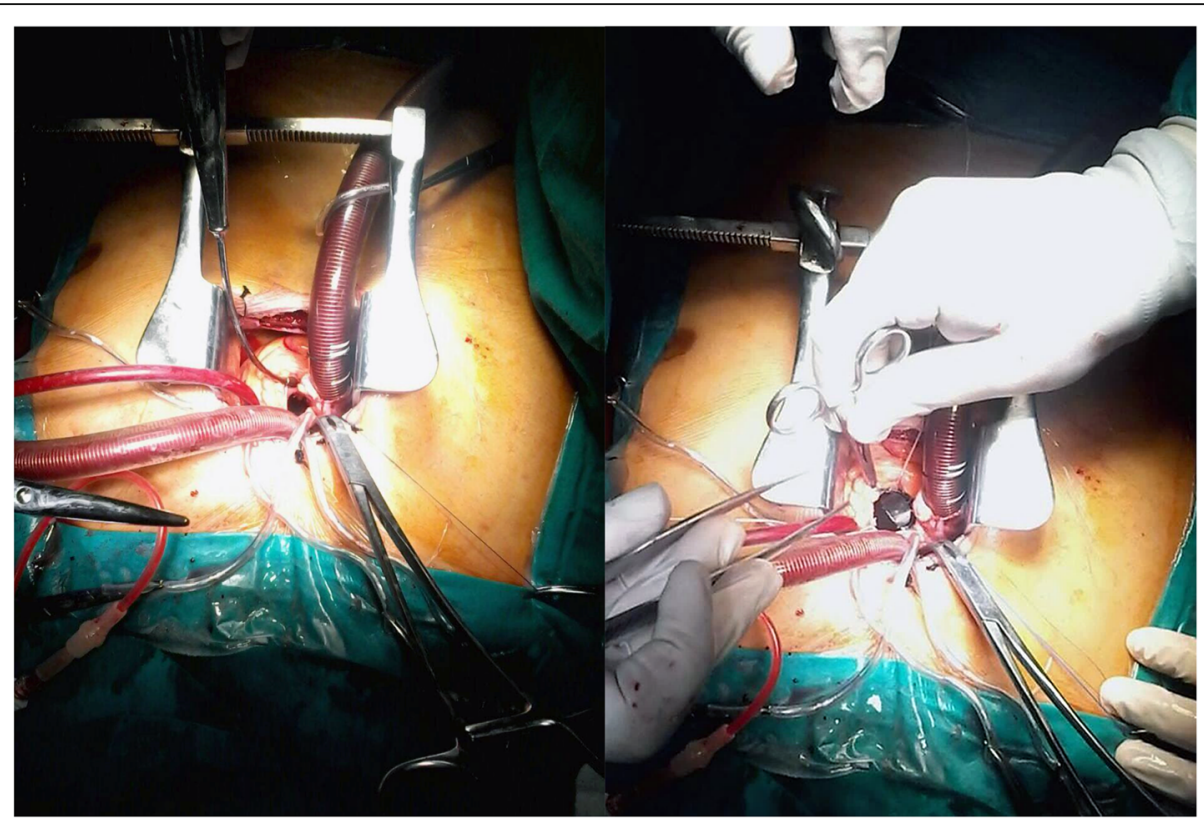

Fig. 1 Intraoperative view of the surgical field with aortic cross-clamp, central cannulation, pulmonary artery vent, and full exposure to the aortic valve with the view of the valve prosthesis in place (right)

The pericardium was retracted to expose the aorta until the origin of the innominate artery. This approach allows the ascending aorta to come forward to obtain better exposure. Two concentric 2-0 polypropylene purse-string sutures were taken at the anterolateral aspect of the ascending aorta as high as possible (Fig. 1).

The cardioplegia cannula was inserted to maintain the least distance of $1 \mathrm{~cm}$ between the cross-clamp and the aortic cannulation site.

Cardiopulmonary bypass was instituted after systemic heparinization using central aortic and dual-stage venous cannulation in all cases. Induced systemic arterial hypotension < $90 \mathrm{mmHg}$ was required during cannulation.

Myocardial protection was established using a standard central aortic cross-clamp followed by antegrade aortic root or direct coronary cold cardioplegia with moderate systemic hypothermia $28-32{ }^{\circ} \mathrm{C}$. Venting was performed via catheterizing the right superior pulmonary vein or the pulmonary artery. The rest of SAVR was completed as the standard of care after evaluation of the valve pathology using an appropriately sized bioprosthesis or mechanical aortic valve prosthesis.

Before weaning off bypass, a single subxiphoid chest drain was used from a $1 \mathrm{~cm}$ transverse skin incision and

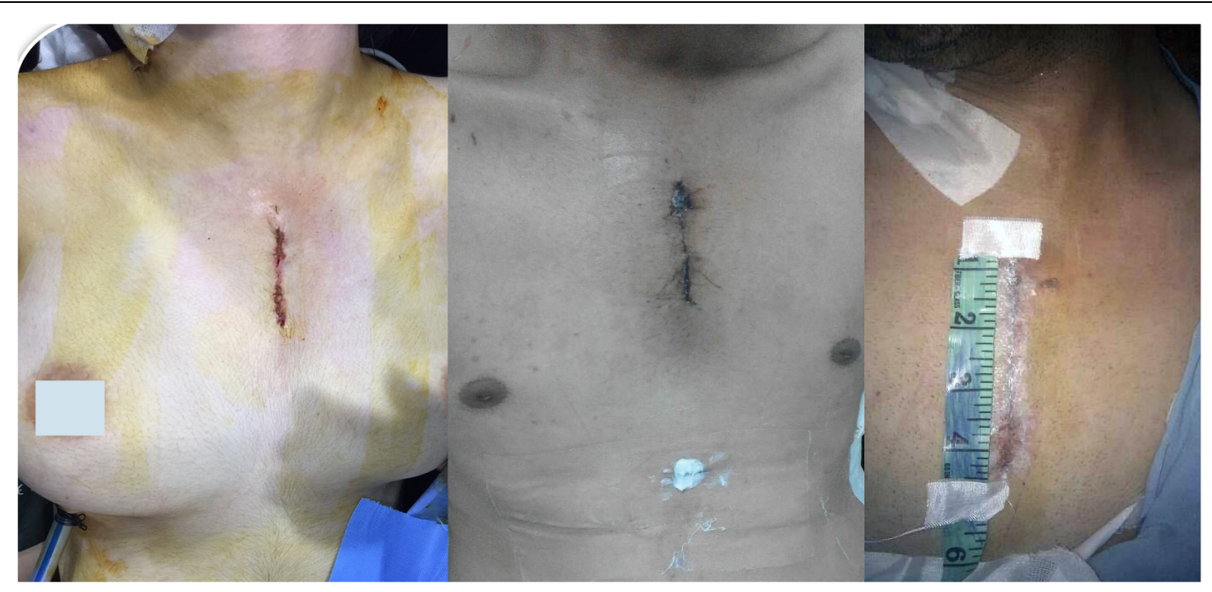

Fig. 2 The incision for minimally invasive aortic valve replacement 
inserted through the pericardial cavity. In the case of pleural collection, a separate intercostal drain was inserted if needed (Fig. 2).

Following hemostasis, 4 to 6 stainless steel wires were used for closure of FS, while only 2 wires were used in the MS group.

\section{Study endpoints}

The primary endpoint was postoperative mechanical ventilation (MV) and intensive care stay times (in hours). The secondary endpoints were cross-clamp (CCT), cumulative cardiopulmonary bypass time (CBP), pain score after $12 \mathrm{~h}$ (1-10), all-cause mortality, need for transfusion, cost analysis, and major adverse cardiac events (MACE) [5], which is a composite endpoint including cardiac death, myocardial infarction, secondary intervention/reoperation, ventricular arrhythmia, renal failure or multi-organ failure, and outcome after 2 years of follow-up. Total cost breakdown was logged for all patients conferring the institutions' financial catalogs.

\section{Statistical analysis}

Based on a pilot study of 10 patients of each technique, the mean hours of ICU stays were found to be $35.7 \pm$ $16.5 \mathrm{~h}$ in MS versus $45.87 \pm 36.78 \mathrm{~h}$ in FS. The least significant total sample size of 94 patients was calculated to have a study power of $85 \%$ with an alpha error of $5 \%$, a beta error of $15 \%$, and an effect size of (Hedges' $\mathrm{g}=$ $(45.87-35.7) / 29.520274=0.344509)$.

A total of 112 patients were enrolled, of which 102 patients met eligibility criteria. The data were tabulated and analyzed using the IBM SPSS software version 22.0 (IBM Inc., Chicago, IL, USA). Qualitative data were described using numbers and percents. Continuous variables were assessed for normality; normal variables were reported as mean \pm standard deviation (SD), while non-normal variables were reported as the median and interquartile range (IQR).

A comparison between categorical variables was performed using the chi-square test ( $\mathrm{x}^{2}$-test). When more than $20 \%$ of the cells have expected count less than 5 , correction for chi-square was conducted using Fisher's exact test or Monte Carlo correction. Confidence intervals $(95 \% \mathrm{CI})$ were calculated. For normally distributed data, a comparison between two independent populations was made using the independent $t$ test. For abnormally distributed data, we used the Mann-Whitney $Z$ test and Wilcoxon signed-ranks tests.

\section{Results}

Our study was conducted on 112 patients, who were operated for aortic valve replacement during the study period, and divided into 2 groups. Fifty-two patients were operated through inverted $\mathrm{T}$-shaped mini- sternotomy (MS); sixty-two patients were operated through a full median sternotomy (FS).

\section{Demographic data}

The baseline characteristics of our sample are mentioned in Tables 1 and 2. There was no significant difference between both groups regarding the preoperative patients' characteristics including age, gender, hypertension, hypercholesterolemia, diabetes mellitus, creatinine clearance, chronic obstructive pulmonary disease, preoperative renal dialysis, extra-cardiac arteriopathy, preoperative stroke, New York Heart Association (NYHA) class, preoperative $\mathrm{EF} \%$, preoperative angina, recent infarction, old infarction, urgency of the operation, preoperative EuroSCORE, valve lesion, valve pathology, preoperative atrial fibrillation, preoperative complete heart block, and preoperative hemoglobin level.

Patients who underwent MIAVR had less body mass index (BMI) than the FS group ( $p=0.002$ and 0.001 , respectively). We included a 54-year-old female morbidly obese diabetic and a hypertensive patient who had a BMI of $60.2 \mathrm{~kg} / \mathrm{m}^{2}$ (obesity class III) who underwent isolate AVR using MS (Fig. 3).

\section{Operative and postoperative outcomes}

All patients were operated for aortic valve replacement. Operative outcomes are mentioned in Table 3.

MS exhibited a significantly less skin incision length of $5.82 \pm 0.67 \mathrm{~cm}$ versus $27.52 \pm 2.71 \mathrm{~cm}(p=0.001)$. No significant differences were observed as regards CCT and $\mathrm{CPB}$ times among both groups $(p=0.58$ and 0.66$)$.

Patients were monitored postoperatively as the standard of care. Postoperative complications are compared in Table 4. In the FS group, $68.3 \%$ had severe postoperative pain, compared with MS group, where $92 \%$ experienced mild pain at the visual analog scale at 12 postoperative hours. MS significantly reduced MV time $(6.18 \pm 1.86$ versus $10.68 \pm 12.78 \mathrm{~h}, p=0.035)$, ICU stay (33.27 \pm 19.75 versus $49.42 \pm 47.1 \mathrm{~h}, p=0.037)$, and blood transfusions (1.18 \pm 0.89 versus $1.7 \pm 0.97$ units, $p=0.002)$. MS patients exhibited fewer pulmonary complications (pneumonia, reintubation, and pleural effusions) $(p<$ $0.001)$, less superficial wound infections $(p=0.048)$, and no deep sternotomy wound infection (DSWI).

Resternotomy was indicated for bleeding in 7 patients. MS group showed less incidence of postoperative hemopericardium but without statistical significance. Four patients required sternal rewiring. A total all-cause operative mortality of 5 cases was reported (4.46\%).

MS group had 2 mortalities; the first was a 58-year-old female diagnosed with severe aortic stenosis complicated with congestive heart failure (LVEF 42\%). She was admitted to ICU on moderate inotropic support with low cardiac output, and mortality occurred on the 
Table 1 Baseline clinical characteristics for the studied sample

\begin{tabular}{|c|c|c|c|c|c|c|}
\hline \multirow{3}{*}{$\overline{\text { Age (years) }}$} & & \multicolumn{2}{|c|}{ Mini-sternotomy (MS) $(n=50)$} & \multicolumn{2}{|c|}{ Full sternotomy (FS) $(n=52)$} & \multirow{2}{*}{$\frac{p}{0.72}$} \\
\hline & $(\text { Mean } \pm \text { SD) })^{a}$ & $41.1 \pm 10.3$ & & 46.4 & & \\
\hline & Min, $\max$ & 28,62 & & 25,7 & & \\
\hline Gender & Male & 37 & (74) & 33 & $(63.5)$ & 0.29 \\
\hline Weight (kg) & $($ Mean \pm SD) & $78.1 \pm 20.4$ & & 89.9 & & 0.02 \\
\hline Height (cm) & $($ Mean \pm SD) & $171.2 \pm 10.3$ & & 171.4 & & 0.49 \\
\hline BMI $\left(\mathrm{kg} / \mathrm{m}^{2}\right)$ & $($ Mean \pm SD) & $26.98 \pm 6.7$ & & 31.1 & & 0.001 \\
\hline BSA & $($ Mean \pm SD) & $1.88 \pm 0.24$ & & 2.02 & & 0.001 \\
\hline \multirow[t]{4}{*}{ Angina status } & $\operatorname{CCS} 1$ & 18 & $(36.0)$ & 7 & $(13.5)$ & $<0.001$ \\
\hline & $\operatorname{CCS} 2$ & 24 & $(48.0)$ & 11 & $(21.2)$ & \\
\hline & $\operatorname{ccs} 3$ & 8 & (16.0) & 33 & $(63.5)$ & \\
\hline & $\operatorname{cCS} 4$ & 0 & $(0.0)$ & 1 & (1.9) & \\
\hline \multirow[t]{4}{*}{ Dyspnea status } & NYHA 1 & 5 & (10) & 0 & (0) & \\
\hline & NYHA 2 & 28 & (56) & 8 & $(15.4)$ & $<0.001$ \\
\hline & NYHA 3 & 17 & (34) & 42 & (80.8) & \\
\hline & NYHA 4 & 0 & (0) & 2 & (3.8) & \\
\hline EurOSCORE \% & $($ Mean \pm SD) & $1.99 \pm 1.43$ & & 2.05 & & 0.64 \\
\hline \multirow[t]{2}{*}{ Congestive cardiac failure } & Never & 50 & (100) & 50 & $(96.2)$ & 0.49 \\
\hline & Past & 0 & (0) & 2 & (3.8) & \\
\hline \multirow[t]{3}{*}{ Diabetes mellitus ${ }^{\mathrm{b}}$} & Diet control & 0 & (0) & 1 & (1.9) & 0.76 \\
\hline & Oral therapy & 3 & (6) & 3 & (5.8) & \\
\hline & Insulin & 2 & (4) & 3 & (5.8) & \\
\hline Hypertension & & 11 & (22) & 18 & (34.6) & 0.21 \\
\hline Hypothyroidism & & 0 & (0) & 1 & (1.9) & 0.24 \\
\hline Dyslipidemia & & 2 & (4) & 7 & $(13.5)$ & 0.14 \\
\hline \multirow[t]{2}{*}{ Smoking } & Ex-smoker & 9 & (18) & 14 & $(26.9)$ & 0.53 \\
\hline & Active smoker & 4 & (8) & 3 & $(5.7)$ & \\
\hline Gl tract & Liver dysfunction & 1 & (2) & 1 & (1.9) & 1 \\
\hline Renal & $\mathrm{Cr}>200 \mathrm{umol} / \mathrm{l}$ & 0 & (0) & 3 & (5.8) & 0.24 \\
\hline \multirow[t]{2}{*}{ Respiratory } & Asthma & 1 & (2) & 0 & (0) & 0.49 \\
\hline & COPD & 1 & (2) & 1 & (1.9) & \\
\hline CVD & Stroke & 1 & (2) & 1 & (1.9) & 1 \\
\hline PVD & Arteriopathy & 1 & (2) & 2 & (3.8) & 1 \\
\hline Preoperative arrhythmia & Atrial fibrillation (AF) & 1 & (2) & 1 & (1.9) & 1 \\
\hline \multirow[t]{2}{*}{ Hemoglobin } & $($ Mean \pm SD) & $13.5 \pm 1.37$ & & 13.24 & & 0.35 \\
\hline & Min, max & $10.2,16.2$ & & $9.8,1$ & & \\
\hline \multirow[t]{2}{*}{ S. creatinine (mg/dl) } & $($ Mean \pm SD) & $0.98 \pm 0.39$ & & 0.84 & & 0.09 \\
\hline & Min, max & $0.5,3$ & & $0.2,1$ & & \\
\hline Creatinine clearance (ml/min) & $($ Mean \pm SD) & $69.2 \pm 22.63$ & & 77.72 & & 0.37 \\
\hline S. bilirubin & $($ Mean \pm SD) & $0.74 \pm 0.37$ & & 0.48 & & 0.13 \\
\hline Coronary angiography & & 12 & (24) & 42 & (80.8) & $<0.001$ \\
\hline
\end{tabular}

$B M I$ body mass index; $C O P D$ chronic obstructive pulmonary disease; $M I$ myocardial infarction; $P V D$ peripheral vascular disease (or extracardiac arteriopathy) as indicated by claudication, amputation for arterial insufficiency, aortoiliac occlusive disease reconstruction, peripheral vascular bypass surgery, angioplasty or stent, or documented abdominal aortic aneurysm; EuroSCORE European System for Cardiac Operative Risk Evaluation; CVD cerebrovascular disease; PVD peripheral vascular disease or extracardiac arteriopathy

${ }^{a}$ The values are the number of patients with the percentage in brackets or the mean \pm standard deviation or the median and interquartile range in brackets ${ }^{\mathrm{b}}$ Basal serum glucose $>200 \mathrm{mg} / \mathrm{dl}$ at three consecutive measurements before surgery 
Table 2 Echocardiographic data

\begin{tabular}{|c|c|c|c|c|c|c|}
\hline & & Mini-sternot & $(\mathrm{MS})(n=50)$ & Full sternotc & $y(F S)(n=52)$ & $p$ \\
\hline \multirow[t]{2}{*}{ LVEF (\%) } & Fair $(30-49 \%)^{\#}$ & 5 & (10) & 7 & $(13.5)$ & 0.58 \\
\hline & Good (> 50\%) & 45 & (90) & 45 & $(86.5)$ & \\
\hline Aortic stenosis & $n(\%)$ & 36 & (72) & 45 & $(86.5)$ & 0.32 \\
\hline Aortic valve area $\left(\mathrm{cm}^{2}\right)$ & $($ Mean \pm SD) & $1.24 \pm 0.37$ & & $1.15 \pm 0.53$ & & 0.72 \\
\hline Preoperative peak $\mathrm{Gr}$. on the valve $(\mathrm{mmHg})$ & (Mean \pm SD) & $71 \pm 19.7$ & & $74.7 \pm 23.7$ & & 0.38 \\
\hline Preoperative mean $\mathrm{Gr}$. on the valve ( $\mathrm{mmHg}$ ) & $($ Mean \pm SD) & $43 \pm 12.5$ & & $47 \pm 17.9$ & & 0.68 \\
\hline \multirow[t]{4}{*}{ Reason for operation } & Severe valve pathology & 22 & (44) & 0 & (0) & $<0.001$ \\
\hline & Anatomical & 25 & (50) & 46 & $(88.5)$ & \\
\hline & Angina & 2 & (4) & 5 & (9.6) & \\
\hline & Unknown & 1 & (2) & 1 & (1.9) & \\
\hline
\end{tabular}

LVEF left ventricular ejection fraction, $\mathrm{Gr}$ gradient, $\mathrm{mmHg}$ millimeters of mercury

${ }^{\#}$ Cases with LVEF $<40 \%$ were excluded as per exclusion criteria

second postoperative day. The second case was a 48year-old male with aortic stenosis who was discharged to the ward following the third postoperative day with a stable condition, and mortality was encountered on the eighth postoperative day.

FS group had 3 mortality cases; the first was a 71-yearold male with severe aortic stenosis and LVEF of $40 \%$. Low cardiac output and LVEF of $32 \%$ were encountered, and mortality occurred on the eighth postoperative day. The second case was a 65-year-old obese male smoker who was diagnosed with severe aortic regurgitation and impaired LVEF of $42 \%$. Mortality was recorded following prolonged mechanical ventilation because of chest infection and sepsis. The third case was a 48-year-old nonsmoker diabetic female who had delayed recovery requiring reintubation and mechanical ventilation. She developed right-sided pneumonia. Sputum culture yielded Klebsiella species. She developed ventricular fibrillation for which she received cardioversion. Mortality was declared on the eighth postoperative day.

One late mortality was recorded upon follow-up of a 58-year-old female with a good left ventricular function who underwent SAVR using the MS approach 1 year following surgery due to cardiac arrest at home without
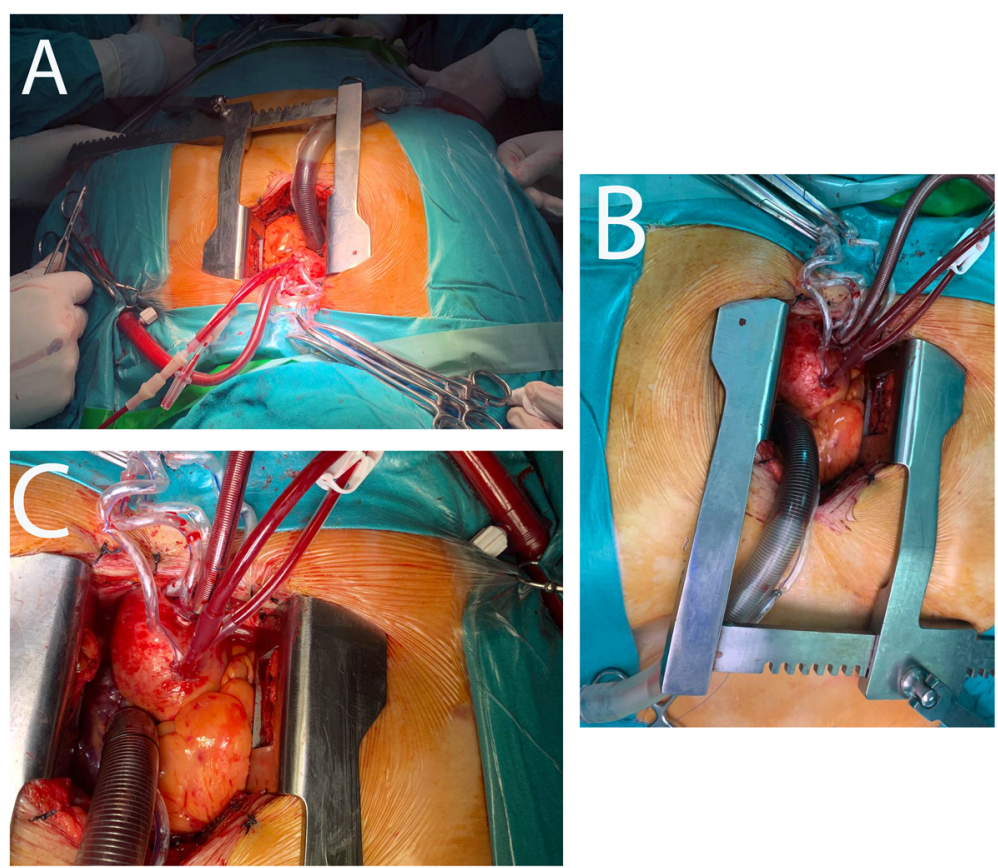

Fig. 3 Exposure for minimally invasive aortic valve replacement in a morbidly obese female patient of BMl=60.2 kg/m² 
Table 3 Operative data for the studied sample

\begin{tabular}{|c|c|c|c|c|c|c|}
\hline \multirow{2}{*}{ Length of skin incision (cm) } & \multirow[b]{2}{*}{ (Mean \pm SD) } & \multicolumn{2}{|c|}{ Mini-sternotomy (MS) $(n=50)$} & \multicolumn{2}{|c|}{ Full sternotomy (FS) $(n=52)$} & \multirow{2}{*}{$\frac{p}{0.001}$} \\
\hline & & $5.82 \pm 0.67$ & & 27.6 & & \\
\hline \multirow[t]{2}{*}{ Implant type } & Mechanical & 38 & (76) & 28 & $(53.8)$ & 0.16 \\
\hline & Biological & 12 & (24) & 24 & $(46.2)$ & \\
\hline \multirow[t]{5}{*}{ Implant size (mm) } & 19 & 4 & (8) & 9 & $(17.3)$ & 0.31 \\
\hline & 21 & 20 & (40) & 23 & $(44.2)$ & \\
\hline & 23 & 16 & (32) & 16 & $(30.8)$ & \\
\hline & 25 & 8 & (16) & 3 & (5.8) & \\
\hline & 27 & 2 & (4) & 1 & $(0.2)$ & \\
\hline \multirow[t]{2}{*}{ Cumulative bypass time (min) } & $($ Mean \pm SD) & $91.87 \pm 34.41$ & & 94.9 & & 0.66 \\
\hline & Min, max & 43,180 & & 50,2 & & \\
\hline \multirow[t]{2}{*}{ Cumulative cross-clamp time (min) } & Mean \pm SD & $68.88 \pm 29.63$ & & 65.78 & & 0.58 \\
\hline & Min, max & $70.2,35$ & & 30,1 & & \\
\hline \multirow[t]{2}{*}{ Minimum core temperature $\left({ }^{\circ} \mathrm{C}\right)$} & Mean \pm SD & $31.02 \pm 2.39$ & & 30.47 & & 0.14 \\
\hline & Min, max & 31,27 & & 27,3 & & \\
\hline \multirow[t]{4}{*}{ Inotropic support } & High & 0 & (0) & 3 & (5.7) & 0.445 \\
\hline & Moderate & 1 & (2) & 2 & (3.8) & \\
\hline & Minimal & 24 & (48) & 26 & (50) & \\
\hline & None & 25 & (50) & 21 & $(38.7)$ & \\
\hline
\end{tabular}

$\mathrm{Cm}$ centimeters, $\mathrm{mm}$ millimeters, $S D$ standard deviation, ${ }^{\circ} \mathrm{C}$ degree celcius

prior symptoms. None of the mortality cases required reexploration or encountered bleeding.

\section{Cost analysis}

All costs were converted to the corresponding US dollar rate using the inpatient hospital service Consumer Price Index (CPI). The total cost for admission and operation was calculated to discharge (direct cost). Indirect costs for sick leaves were not calculated.

Looking at the breakdown of costs (Table 5), significant cost reduction was recorded favoring MS approach as regards blood product cost $(17.75 \%, p<0.0001)$, ICU stay $(34.48 \%, p<0.0001)$, ward admission $(15.46 \%, p<$ $0.0001)$, and total cost reduction of $\$ 580(9.35 \%, p<$ $0.0001)$.

\section{Discussion}

The lack of stable supplies is the foremost issue limiting us from performing minimally invasive cardiac surgery. Supplies may not be existing at all, or out of stock. As we are a government-funded institution, our supply chain is affected by the assets of the country, which are currently in a poor state.

In order to conquer some of these problems, we have had to espouse groundbreaking stratagems in other resource-poor settings to make our patients' profit splendor of minimally invasive cardiac surgery. Minimal invasive surgery offers benefits more than being only cosmetically appealing. It has comparable results to the conventional FS [1].

Our study was novel in that it utilized itemized facility costs for the procedure for each technique. Our facility divided costs based on direct patient care (including blood, laboratory, theater, room, supplies, therapy, imaging, and miscellaneous costs).

It is clear from multiple studies [3] that antegrade perfusion through the ascending aorta has several advantages as being more physiological and reduces the risk of embolization, iatrogenic aortic dissection, and groin incisions with associated complications. We used the advantage of antegrade perfusion along the minimally invasive SAVR.

A recent Cochrane review [6] encompassed seven trials with 511 participants from 7 countries. The effects of minimally invasive limited upper MS on SAVR as compared with FS were investigated [7-13].

In our study, we found that CBP and CCT times were not significantly different between both techniques. Notably, total operative time was significantly longer in the MS group in our first cases. In accordance with our results, there was no evidence of an increase CBP with AVR performed via an upper MS (mean difference (MD) $3.02 \mathrm{~min}, 95 \% \mathrm{CI}-4.10$, 10.14; participants $=311$; studies $=5$; low quality). There was no evidence of an increase in aortic CCT (MD $0.95 \mathrm{~min}, 95 \% \mathrm{CI}-3.45$, 5.35; participants $=391$; studies $=6$; low quality) $[7,9-11,13]$. 
Table 4 Postoperative data

\begin{tabular}{|c|c|c|c|c|c|c|}
\hline \multirow{2}{*}{ ICU and hospital stay } & & \multicolumn{2}{|c|}{$\begin{array}{l}\text { Mini-sternotomy (MS) } \\
(n=50)\end{array}$} & \multicolumn{2}{|c|}{$\begin{array}{l}\text { Full sternotomy (FS) } \\
(n=52)\end{array}$} & \multirow[t]{2}{*}{$p(95 \% \mathrm{Cl})$} \\
\hline & & & & & & \\
\hline \multirow[t]{2}{*}{ Ventilation time (days) } & $($ Mean $\pm S D)$ & $1 \pm 0$ & & 1.17 & & $0.188(-0.417,0.831)$ \\
\hline & Min, max & 1,1 & & 1,6 & & \\
\hline \multirow[t]{2}{*}{ Ventilation time (h) } & $($ Mean $\pm S D)$ & $6.18=$ & & 10.6 & 2.78 & $0.029(-6.63,-1.09)$ \\
\hline & Min, max & 6,18 & & 4,90 & & \\
\hline \multirow[t]{2}{*}{ ICU stay (nights) } & $($ Mean \pm SD $)$ & $1.43=$ & & $2.1 \pm$ & & $0.035(-1.29,-0.492)$ \\
\hline & Min, max & 1,6 & & 4,10 & & \\
\hline \multirow[t]{2}{*}{ ICU stay (h) } & $($ Mean $\pm S D)$ & 33.27 & & 49.4 & & $0.037(-2.659,-0.978)$ \\
\hline & Min, max & 18,12 & & 4,24 & & \\
\hline \multirow[t]{2}{*}{ Postoperative hospital stay (days) } & $($ Mean $\pm S D)$ & $6.95=$ & & 8.34 & & $0.105(-3.156,0.297)$ \\
\hline & Min, max & 2,25 & & 4,13 & & \\
\hline \multicolumn{2}{|l|}{ Postoperative complications } & 5 & (10) & 7 & $(11.3)$ & 0.096 \\
\hline \multirow[t]{2}{*}{ Transfusion number of blood units } & $($ Mean $\pm S D)$ & \multicolumn{2}{|c|}{$1.18 \pm 0.89$} & \multicolumn{2}{|c|}{$1.7 \pm 0.97$} & $0.002(-0.078,-0.244)$ \\
\hline & Min, max & \multicolumn{2}{|l|}{0,4} & \multicolumn{2}{|c|}{0,5} & \\
\hline Need for transfusion (cases) & $n(\%)$ & 34 & (68) & 38 & $(73.1)$ & 0.052 \\
\hline Resternotomy & Bleeding/tamponade & 2 & (4) & 5 & (9.6) & 0.437 \\
\hline Sternal resuturing & & 1 & (2) & 3 & (5.8) & 0.627 \\
\hline \multirow[t]{3}{*}{ Arrhythmia } & Ventricular fibrillation & 0 & (0) & 3 & (5.8) & 0.014 \\
\hline & Supraventricular tachycardia & 0 & (0) & 0 & $(0)$ & \\
\hline & Permanent block—pace & 2 & (4) & 1 & $(0.2)$ & \\
\hline \multirow[t]{2}{*}{ Pulmonary complications } & Pleural effusion and drainage & 1 & (2) & 2 & (3.8) & $<0.001$ \\
\hline & Reintubation/ventilation & 0 & (0) & 4 & $(7.7)$ & \\
\hline \multirow[t]{2}{*}{ Neurological complications } & Delayed recovery/stroke/other & 0 & (0) & 1 & (1.9) & 0.138 \\
\hline & TIA & 0 & (0) & 1 & $(1.9)$ & \\
\hline \multirow[t]{2}{*}{ Infective complications } & Superficial wound infection & 1 & (2) & 5 & (9.6) & $0.048(-0.194,-0.179)$ \\
\hline & DSWI & 0 & (0) & 1 & $(1.9)$ & \\
\hline Renal complications & $\mathrm{Cr}>3$ & 1 & (2) & 3 & $(5.7)$ & 0.26 \\
\hline Gl complications & Upper Gl bleeding & 0 & (0) & 4 & $(7.7)$ & 0.22 \\
\hline Multi-organ failure & $n(\%)$ & 0 & (0) & 2 & (3.8) & 0.34 \\
\hline \multirow[t]{2}{*}{ Discharged to } & Died in hospital & 2 & (4) & 3 & $(5.7)$ & 0.67 \\
\hline & Home & 48 & (96) & 49 & $(94.2)$ & \\
\hline \multirow[t]{3}{*}{ Status at 2 years of follow-up } & Alive & 47 & (94) & 49 & $(94.2)$ & 0.55 \\
\hline & Died in hospital & 2 & (4) & 3 & $(5.7)$ & \\
\hline & Died & 1 & (2) & 0 & (0) & \\
\hline
\end{tabular}

Unlike our study, none of the included studies in this review reported major adverse cardiac and cerebrovascular events as a composite endpoint. There was no evidence of any effect of upper MS on mortality versus FS (risk ratio (RR) 1.01, 95\% (CI) 0.36 to 2.82; participants $=511$; studies $=7$; moderate quality) $[9,12]$.

We calculated the duration of postoperative MV from the moment of intensive care unit admission to the moment of extubation of the endotracheal tube. We observed a shorter period of postoperative MV in the MS group compared with the FS group. We contemplate that these results were due to the upheld integrity of the thoracic cage, expressly the lower costal margin, which helps in the conservation of the respiratory mechanics. The smaller incision can be convoyed with less pain and easier respiratory movements.

The results of mini-sternotomy are promising regarding decreasing $\mathrm{MV}$ and ICU stay, which will have a 
Table 5 Mean total cost breakdown

\begin{tabular}{|c|c|c|c|c|}
\hline & Mini-sternotomy (MS) $(n=50)$ & Full sternotomy (FS) $(n=52)$ & Difference mean (\%) $(95 \% \mathrm{Cl})$ & $p$ \\
\hline \multicolumn{5}{|l|}{ Operative costs } \\
\hline Theater admission & $\$ 72.58$ & $\$ 72.58$ & & NS \\
\hline Oxygenator and tubing & $\$ 362.89$ & $\$ 362.89$ & & NS \\
\hline Cannulae (aortic and two stage venous) & $\$ 181.44$ & $\$ 181.44$ & & NS \\
\hline Drainage system & $\$ 38.72$ & $\$ 38.72$ & & NS \\
\hline Suturing material & $\$ 211.67$ & $\$ 211.67$ & & NS \\
\hline Sternal wire & $\$ 60.42$ & $\$ 90.42$ & & NS \\
\hline Mechanical prosthesis & $\$ 604.78$ & $\$ 604.78$ & & NS \\
\hline Biological prosthesis & $\$ 1632.95$ & $\$ 1632.95$ & & NS \\
\hline Blood & $\$ 241.92$ & $\$ 294.32$ & $\$ 52.08(17.75 \%)(33.33,70.83)$ & $<0.0001$ \\
\hline Operating team fees & $\$ 2419.2$ & $\$ 2419.2$ & & NS \\
\hline \multicolumn{5}{|l|}{ Postoperative costs } \\
\hline ICU stay and pharmacy & $\$ 529.77$ & $\$ 809.75$ & $\$ 279.23(34.48 \%)(223.86,334.61)$ & $<0.0001$ \\
\hline Ventilator & $\$ 78.64$ & $\$ 78.64$ & & NS \\
\hline Ward & $\$ 349.6$ & $\$ 413.32$ & $\$ 63.72(15.46 \%)(35.47,91.98)$ & $<0.0001$ \\
\hline Imaging & $\$ 54.25$ & $\$ 62.54$ & $\$ 8.29(13.25 \%)(-3.975,20.56)$ & 0.1832 \\
\hline Laboratory & $\$ 415.75$ & $\$ 498.13$ & $\$ 82.38$ (16.57\%) $(54.08,110.69)$ & $<0.0001$ \\
\hline Total cost (mechanical) & $\$ 5621.63$ & $\$ 6201.76$ & $580.13(9.35 \%)(351.08,649.18)$ & $<0.0001$ \\
\hline Total cost (biological) & $\$ 6649.81$ & $\$ 7229.93$ & 580.12 (8.02\%) (281.61, 719.38) & 0.0001 \\
\hline
\end{tabular}

NS non-significant statistically at $p>0.05$

positive economic impact. Upper inverted $\mathrm{T}$-shaped hemi-sternotomy maintains the integrity of the chest wall, which will improve the chest wall mechanics and facilitate early weaning and ambulation. This is a major advantage of MS over FS other than the cosmetic results and should be the primary target of the approach. In paradox, literature reported no significant effect on $\mathrm{MV}$ times (MD - 1.12 h, 95\% CI - 3.43 to 1.19; participants $=297$; studies $=5$; low quality) $[7,8,10-13]$.

We observed significantly shorter ICU stay a slightly shorter, but non-significant, duration of the postoperative hospital stay in the MS group. In the literature, there was a small reduction in length of intensive care unit stays as a result of the MS (MD was - 0.57 days, $95 \% \mathrm{CI}-0.93$ to -0.20 ; participants $=297$; studies $=5$; low quality) [9]. However, there was no evidence of an effect on length of hospital stay (MD - 1.31 days, 95\% $\mathrm{CI}-2.63,0.01$; participants $=297$; studies $=5 ; \mathrm{I} 2=89 \%$; very low quality) $[7,8,10,1113]$.

In agreement to our results, postoperative blood loss was lower in MS (MD - $158.00 \mathrm{ml}, 95 \% \mathrm{CI}-303.24$ to - 12.76; participants $=297$; studies $=5$; moderate quality). The literature did not attest a drop in DSWI (RR 0.71, 95\% CI 0.22 to 2.30; participants = 511; studies $=7$; moderate quality) or re-exploration (risk ratio (RR) 1.01, $95 \%$ CI 0.48 to 2.13 ; participants $=511$; studies $=7$; moderate quality) $[5,6]$.
Dissimilar to our results, a review reported no change in pain scores by upper MS (standardized mean difference $(\mathrm{SMD})-0.33,95 \% \mathrm{CI}-0.85$ to 0.20 ; participants = 197 ; studies $=3 ; \mathrm{I} 2=70 \%$; very low quality) $[8,10,11]$.

Our study did not address pulmonary function test as an endpoint. The literature reported a small increase in postoperative pulmonary function tests with MS (MD 1.98\% predicted FEV1, 95\% CI 0.62 to 3.33; participants = 257; studies $=4 ; \mathrm{I} 2=28 \%$; low quality) [6].

MS approach did not reduce perioperative atrial fibrillation (AF) compared with FS (RR 0.60, 95\% CI 0.07 to 4.89; participants $=240$; studies $=3$; moderate quality). All of our patients, except one, were having sinus rhythm [6].

None of the included studies in the Cochrane review reported cost analyses. This was a major advantage of the present study.

More recently, the MAVRIC (manubrium-limited mini-sternotomy versus conventional sternotomy for aortic valve replacement trial) [14] a single-center, single-blind randomized study that compared AVR via manubrium-limited mini-sternotomy using a $5-$ to $7-\mathrm{cm}$ midline incision (intervention) and conventional FS and had postoperative red cell transfusion as the primary outcome. MIS was allied to higher CBP time and reduced drain losses, but this difference did not translate in a significant reduction in blood transfusion. Additionally, conventional SAVR was found to be more cost- 
effective (MS had a 5.8\% probability of being costeffective) [14].

\section{Study limitations and strength}

There are some imperative shortcomings of this study. The prospective cohort study is non-randomized. The sample size was small, and the calculation was based on a single outcome of the ICU stay. Other vital outcomes were mentioned as secondary outcomes. Despite the orientation of all surgical teams with both approaches, several surgeons still preferred a traditional FS, so there might have been some selection bias as allocation was according to surgeons' preference. The unequal number of groups was another issue. However, the groups were almost matched demographically. Despite the FS group had of higher BMI, we included cases with morbid obesity in the mini-sternotomy group up to $158 \mathrm{~kg} / \mathrm{BMI} 60.2$ $\mathrm{kg} / \mathrm{m}^{2}$ without impact on postoperative outcomes. The study might be underpowered to detect significance in the difference in low-frequency events. This may or may not have affected some of the important outcomes such as pulmonary or neurological complications but not the ICU stay. Although we incorporated fast recovery protocols in our ICU, the duration of endotracheal intubation and length of postoperative stay was decided by the individual surgeon. No conversions were recorded.

Aortic surgery via mini-sternotomy has already been done in many centers worldwide, this study introduced MS at our centers with limited resources and achieved excellent outcomes with some benefits.

Evidently, the benefit of MS over FS is still debated, and some outcomes of each are well established as cosmetic superiority, bleeding reduction, postoperative pain, $\mathrm{MV}$, and ICU stays as well as reduced pulmonary complications. In light of low resources, patients in developing countries still should benefit the splendor of MIAVR that has verified reduced budgets.

\section{Conclusions}

With the lack of logistics and equipment in developing countries, MIAVR carries a significant reduction of blood product use, respiratory problems, pain, resource utilization, and cosmesis over FS without additional equipment. MIAVR using MS is feasible, with a rapid learning curve, in developing centers.

\footnotetext{
Abbreviations

BMI: Body mass index; CCT: Cumulative cross-clamp time; Cl: Confidence interval; CPB: Cardiopulmonary bypass time; CPI: Consumer price index; DSWI: Deep sternotomy wound infection; EF\%: Ejection fraction percentage; FS: Full sternotomy; ICU: Intensive care unit; MACEs: Major adverse cardiac events; MAVRIC: Manubrium-limited mini-sternotomy versus conventional sternotomy for aortic valve replacement trial; MIAVR: Minimally-invasive approaches to aortic valve surgery; MS: Mini-sternotomy/hemi-sternotomy; MV: Mechanical ventilation; NYHA: New York Heart Association class; RAT: Right anterior thoracotomy; RR: Risk ratio; SAVR: Surgical aortic valve
}

replacement; SD: Standard deviation; SMD/MD: Standardized mean difference

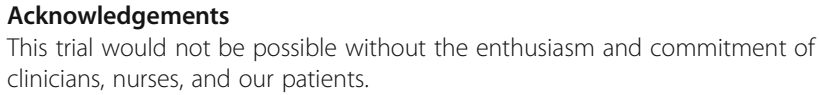

\section{Authors' contributions}

Both authors contributed equally in conceptualization, operating cases. MS presented the data in several international meetings and revised the manuscript; HB performed statistical analysis and writing of the manuscript with MS. All authors have read and approved the final manuscript.

\section{Funding}

No funding was received, self-funded.

\section{Availability of data and materials}

The datasets used and/or analyzed during the current study available from the corresponding author on reasonable request.

\section{Ethics approval and consent to participate}

We followed the declaration of Helsinki regarding studies on human subjects. Approvals of the institutional research boards of Mansoura University Institutional Research Board and Nasser Institute for Research and Treatment Board were obtained (MUIRB-78-715-23) - (NIRT-23/4/15). Patients were informed and signed written consents for participation in the study and operation.

\section{Consent for publication}

Patients were informed and signed written consents for publishing of clinical data.

\section{Competing interests}

The authors declare that they have no competing interests.

\section{Author details}

${ }^{1}$ Department of Cardiothoracic Surgery, Faculty of Medicine, Mansoura University Hospitals, D17, F5. 60, El Gomhoria Street, Qism 2, Mansoura, Dakahlia 35516, Egypt. ${ }^{2}$ Department of Cardiothoracic Surgery, Egypt Ministry of Health and Population, Nasser Institute for Research and Treatment, Cairo, Egypt. ${ }^{3}$ Department of Cardiothoracic Surgery, Egypt Ministry of Health and Population, Alexandria Directorate, Alexandria, Egypt.

Received: 23 November 2019 Accepted: 6 February 2020

Published online: 06 March 2020

\section{References}

1. Glauber M, Ferrarini M, Miceli A (2015) Minimally invasive aortic valve surgery: state of the art and future directions. Ann Cardiothorac Surg 4(1): 26-32

2. Hancock HC, Maier RH, Kasim AS et al (2019) Mini-sternotomy versus conventional sternotomy for aortic valve replacement. J Am Coll Cardiol 73(19):2491-2492

3. Murzi M, Glauber M (2015) Central versus femoral cannulation during minimally invasive aortic valve replacement. Ann Cardiothoracic Surg 4(1): 59-61

4. World Medical Association (2001) World Medical Association Declaration of Helsinki. Ethical principles for medical research involving human subjects. Bull World Health Organ 79(4):373-374

5. Myles PS (2014) Meaningful outcome measures in cardiac surgery. J Extra Corpor Technol 46(1):23-27

6. Kirmani BH, Jones SG, Malaisrie SC, Chung DA, Williams RJNN (2017) Limited versus full sternotomy for aortic valve replacement (Review). Cochrane Database Syst Rev 4:CD011793

7. Aris A, Camara ML, Montiel J, Delgado LJ, Galan J, Litvan H (1999) Ministernotomy versus median sternotomy for aortic valve replacement: a prospective, randomized study. Ann Thorac Surg 67:1583-1587

8. Bonacchi M, Prifti E, Giunti G, Frati G, Sani G (2002) Does ministernotomy improve postoperative outcome in aortic valve operation? A prospective randomized study. Ann Thorac Surg 73:460-465 
9. Borger MA, Moustafine V, Conradi L, Knosalla C, Richter M, Merk DR et al (2015) A randomized multicenter trial of minimally invasive rapid deployment versus conventional full sternotomy aortic valve replacement. Ann Thorac Surg 99:17-25

10. Calderon J, Richebe P, Guibaud JP, Coiffic A, Branchard O, Asselineau J et al (2009) Prospective randomized study of early pulmonary evaluation of patients scheduled for aortic valve surgery performed by ministernotomy or total median sternotomy. J Cardiothorac Vasc Anesth 23:795-801

11. Dogan S, Dzemali O, Wimmer-Greinecker G, Derra P, Doss M, Khan MF et al (2003) Minimally invasive versus conventional aortic valve replacement: a prospective randomized trial. J Heart Valve Dis 12:76-80

12. Mächler HE, Bergmann P, Anelli-Monti M, Dacar D, Rehak P, Knez I et al (1999) Minimally invasive versus conventional aortic valve operations: a prospective study in 120 patients. Ann Thorac Surg 67:1001-1005

13. Moustafa MA, Abdelsamad AA, Zakaria G, Omarah MM (2007) Minimal vs median sternotomy for aortic valve replacement. Asian Cardiovascular Thoracic Annals 15:472-475

14. Akowuah E, Goodwin TA, Owens WA, Hancock HC, Rebecca Maier R, Kasim A, Mellor A, Khan K, Murphy G, Mason J (2017) Manubrium-limited ministernotomy versus conventional sternotomy for aortic valve replacement (MAVRIC): study protocol for a randomised controlled trial. Trials. 18:46

\section{Publisher's Note}

Springer Nature remains neutral with regard to jurisdictional claims in published maps and institutional affiliations.

\section{Submit your manuscript to a SpringerOpen ${ }^{\circ}$ journal and benefit from:}

- Convenient online submission

- Rigorous peer review

- Open access: articles freely available online

- High visibility within the field

- Retaining the copyright to your article

Submit your next manuscript at $\boldsymbol{\nabla}$ springeropen.com 